ARTICLE

DOI: $10.1038 / s 41467-018-03047-9$

\title{
G-protein $\beta \gamma$ subunits determine grain size through interaction with MADS-domain transcription factors in rice
}

Qian Liu1, Ruixi Han ${ }^{1,2,3}$, Kun Wu ${ }^{1,4}$, Jianqing Zhang1,2, Yafeng $\mathrm{Ye}^{1}$, Shuansuo Wang ${ }^{1}$, Jianfeng Chen ${ }^{1,2}$, Yajun Pan ${ }^{1}, \mathrm{Qi} \mathrm{Li}^{1}$, Xiaopeng $\mathrm{Xu}^{1,5}$, Jiawu Zhou ${ }^{6}$, Dayun Tao ${ }^{6}$, Yuejin $\mathrm{Wu}^{4} \&$ Xiangdong $\mathrm{Fu}^{1,2}$

The simultaneous improvement of grain quality and yield of cereal crops is a major challenge for modern agriculture. Here we show that a rice grain yield quantitative trait locus $q L G Y 3$ encodes a MADS-domain transcription factor OsMADS1, which acts as a key downstream effector of G-protein $\beta \gamma$ dimers. The presence of an alternatively spliced protein OsMADS1gy3 is shown to be associated with formation of long and slender grains, resulting in increases in both grain quality and yield potential of rice. The G $\gamma$ subunits GS3 and DEP1 interact directly with the conserved keratin-like domain of MADS transcription factors, function as cofactors to enhance OsMADS1 transcriptional activity and promote the co-operative transactivation of common target genes, thereby regulating grain size and shape. We also demonstrate that combining OsMADS1gy3 allele with high-yield-associated dep1-1 and gs3 alleles represents an effective strategy for simultaneously improving both the productivity and end-use quality of rice.

\footnotetext{
${ }^{1}$ The State Key Laboratory of Plant Cell and Chromosome Engineering, Institute of Genetics and Developmental Biology, Chinese Academy of Sciences, 100101 Beijing, China. ${ }^{2}$ College of Life Sciences, University of Chinese Academy of Sciences, 100049 Beijing, China. ${ }^{3}$ Development Center for Science and Technology, Ministry of Agriculture, 100122 Beijing, China. ${ }^{4}$ Institute of Technical Biology and Agriculture Engineering, Hefei Institutes of Physical Science, Chinese Academy of Sciences, 230031 Hefei, China. ${ }^{5}$ Root Biology Center, South China Agricultural University, 510642 Guangzhou, China. ${ }^{6}$ Food Crops Research, Institute Yunnan Academy of Agricultural Sciences, 650200 Kunming, China. Qian Liu and Ruixi Han contributed equally to this work. Correspondence and requests for materials should be addressed to X.F. (email: xdfu@genetics.ac.cn)
} 
D espite the genetic improvement of grain yield delivered by the exploitation of semi-dwarfism and heterosis over the past 50 years $^{1-3}$, a substantial increase in grain productivity of the major crops is required to feed a growing world population ${ }^{4}$. The prime breeding target is to increase both grain size and grain number, because they impact both on yield potential and its end-use quality ${ }^{5}$. However, the simultaneous improvement of grain quality and grain yield is a major challenge because of the well-established negative correlation between these two traits ${ }^{6}$, all of which is controlled by quantitative trait loci (QTL) and influenced by environmental changes. Rice is a staple food for nearly one-half the world's population, molecular and genetic basis of main yield components (i.e., tiller numbers per plant, grain numbers per panicle, and 1,000-grain weight) have been extensively investigated in the last decade. Recently, several genes have been shown to control grain size and grain number of rice: Gn1a/OsCKX2 ${ }^{7}$, DENSE AND ERECT PANICLE1 $(D E P 1)^{8-10}$, GNP1/GA20ox $1^{11}$, IPA1/WFP/OsSPL14 ${ }^{12,13}$, and NPT1/OsOTUB1 ${ }^{14}$ regulate panicle architecture and grain number; GS3 ${ }^{15}$, TGW6 ${ }^{16}, G W 8 / O s S P L 16^{5,17}, G W 7 / G L 7^{17,18}$, GLW7/OSSPL13 ${ }^{19}$, $G S 2 / G L 2 / O s G R F 4^{20-22}, G W 2^{23}$, and $G W 5^{24,25}$ regulate grain size and shape. However, the genetic determinants of other QTLs that regulate grain size and number remain poorly understood. Therefore, identification of allelic variations of other genes associated with improvement of grain yield would facilitate the breeding of new high-yielding rice varieties and may be applicable to other crops.

Natural variants of the rice G-protein $\gamma$ subunits $\mathrm{DEP} 1^{8-10}$ and GS3 $3^{15}$ have been shown to boost grain yield, but typically show only a mediocre quality of grains. The nature of variations associated with higher yield potential is also quite different for each of the two $\mathrm{G} \gamma$ subunits ${ }^{26}$. The $g s 3$ loss-of-function allele is associated with the formation of a long and slender grain, resulting in an increase in the size of grains, and the reduction of grain number ${ }^{15}$. In contrast, the dep1-1 gain-of-function allele caused an increase in the number of grains per panicle, but produces smaller grains ${ }^{8,10}$. Indeed, the simultaneous improvement of seed size and number is a major challenge for modern agriculture, because of a genetic trade-off between these two traits that producing more seeds would lead to smaller seeds and vice vers ${ }^{27}$. Grain size and number are inherently connected with floral organ identity and growth, which are tightly regulated by various combinations of MADS-domain transcription factors ${ }^{28}$. In rice, homo- and hetero-dimerization and formation of multiple complex and interaction with other proteins (e.g., transcription factors, kinases, and chromatin modifiers) in higher order complexes have been described, but how the members of these interacting partners execute specific target gene expression is not yet fully understood ${ }^{29}$. In addition, changes in multiple environment factors, including biotic and abiotic stresses affect seed size and number. For example, plants grown in resourcelimited environments may benefit from producing large seeds of small crops, resulting in offspring seedlings capable of survival and growth under fluctuating environments ${ }^{30}$. However, the molecular mechanisms underlying the interplay between floral organ identity specification and growth capacity in response to environmental changes still remain unknown.

Here we show that a quantitative trait locus LGY3 is synonymous with OsMADS1 ${ }^{29}$, which encodes a MADS-domain transcription factor. Our results show that a natural variant of $O s M A D S 1^{l g y 3}$, an alternatively spliced form of OsMADS1, is associated with the formation of a more slender grain and better appearance quality. We demonstrate that the rice G $\beta \gamma$ subunits act as conserved interacting cofactors with OsMADS1. DEP1 and OsMADS1 share common targets in vivo and their physical interaction is important in driving downstream transcriptional expression profiles and in regulating grain size and shape. Our findings reveal a new molecular framework for the control of variation in seed size and number in response to fluctuating environments. More importantly, haplotype analysis of the OsMADS1 gene reveals that the L-204 haplotype involving truncating splice-site mutation is common within $O$. nivara accessions and tropical japonica germplasm, but it does not appear to occur within the elite indica and temperate japonica rice varieties, thus combining the OsMADS1 lgy 3 allele with the high-yielding dep1-1 and gs3 alleles provides a new strategy for simultaneously improving grain quality and yield potential of rice.

\section{Results}

Identification of $q L G Y 3$. The japonica rice variety Wuyunjing7 carrying the dep1-1 allele (hereafter WYJ7-dep1-1) produces more, but smaller grains than its near-isogenic line (NIL) WYJ7$D E P 1^{10}$ (Fig. 1a). A set of 250 recombinant inbred lines (RILs) were developed from the cross between Wuyunjing7 and the American japonica rice variety L-204, which produces long and slender grains, one line (RIL186) carrying the dep1-1 allele formed grains that were bigger than that formed by the WYJ7dep1-1 parent (Fig. 1a). A subsequent QTL analysis revealed the presence of a quantitative trait locus $q L G Y 3$, which was pleiotropically responsible for long-grain and high-yield traits (Fig. 1b). Genetic analysis of $\mathrm{BC}_{4} \mathrm{~F}_{2}$ progenies derived from a crossing of RIL186 with the indica rice variety HJX74 (the recurrent parent) suggested that a semi-dominant qlgy 3 allele from L-204 was associated with the characteristics of both longgrain and high-yield (Supplementary Fig. 1).

OsMADS1 $1^{\text {lgy3 }}$ is associated with long and slender grains. Positional cloning of qlgy 3 was performed by using $\mathrm{BC}_{2} \mathrm{~F}_{2}$ and $\mathrm{BC}_{3} \mathrm{~F}_{2}$ populations developed from the cross between $\mathrm{R} 186$ and Thai cultivar RD23 (the recurrent parent), and candidate region was narrowed to $\mathrm{a} \sim 8.9 \mathrm{Kbp}$ stretch flanked by molecular markers XP22 and XP23 (Fig. 1c). This segment only contains the coding and $3^{\prime}$ untranslated regions of OsMADS1, a gene encoding MADS-domain transcription factor ${ }^{29}$. Sequence analysis indicated that an insertion-deletion polymorphism in the splice site of the intron 7/exon 8 junction was differentiated between L-204 and RD23 (Fig. 1d), resulting in L-204 producing an alternatively spliced protein OsMADS1 19y3 , in which the terminal 37 residues were truncated, and an additional 5 residues were added to its predicted C domain (Fig. 1d). The effect of truncating splice-site mutation was to increase slightly the transcript and protein accumulation, without affecting nuclear localization and its interaction with other MADS-domain proteins ${ }^{31}$ (Supplementary Fig. 2).

A NIL NPB-lgy3 was created in the japonica variety Nipponbare background by introgressing a 274-Kbp segment from L-204 (Supplementary Fig. 3a). The NPB-lgy3 plants formed grains that were longer than those formed by Nipponbare control plants (hereafter NPB-LGY3), but there was little difference with respect to grain width (Supplementary Fig. 3b-d). Furthermore, either length or width of NPB-lgy3 outer epidermal cells was indistinguishable from that of the equivalent cells in NPB-LGY3 (Supplementary Fig. 3e, f). These observations suggested that the $\lg y 3$ allele was correlated with the production of more slender grains as results of an increased cell division in the longitudinal direction, but no changes in cell proliferation and elongation in the transverse direction. The grains formed by the transgenic NPB-LGY3 plants expressing the L-204 lgy 3 cDNA driven either by its native promoter or by the rice Actin promoter were longer than those formed by non-transgenic control plants, as well as 
a

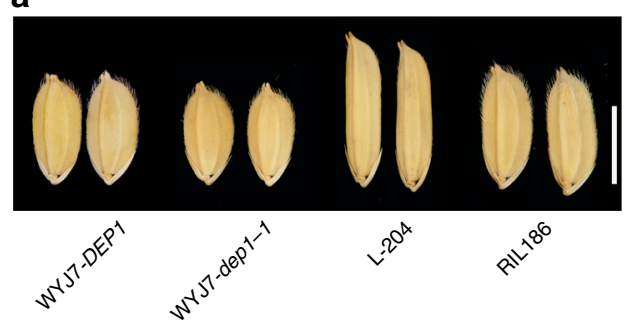

b

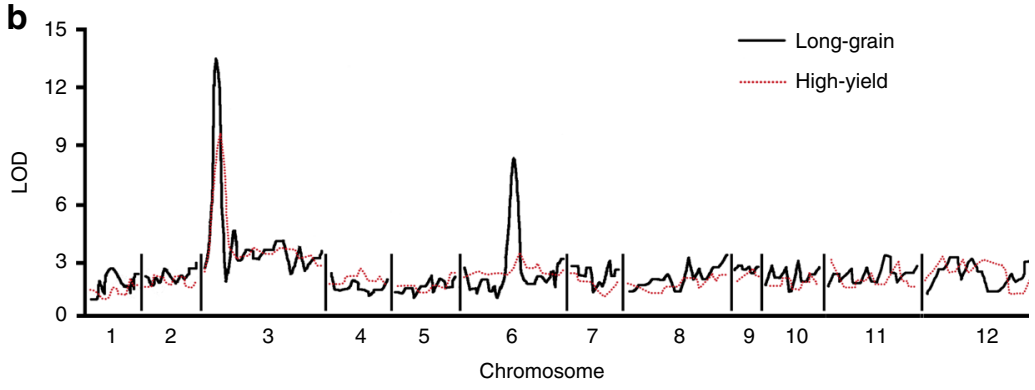

C

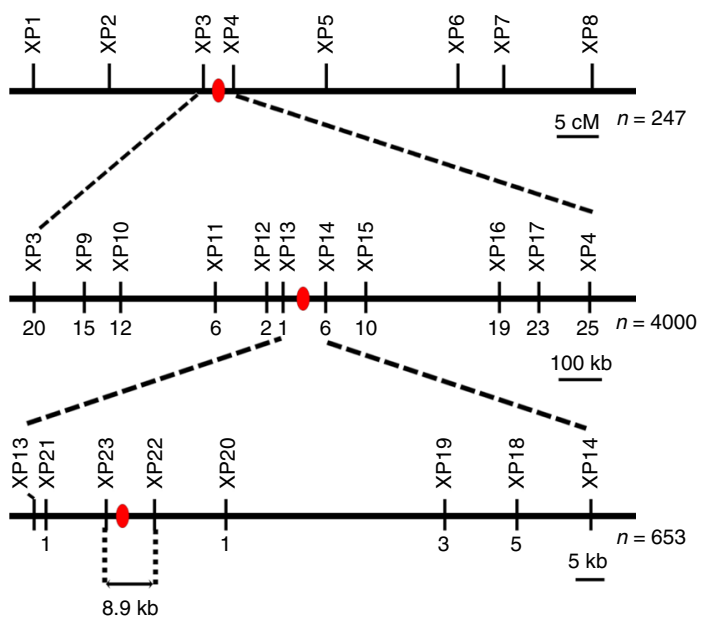

d OsMADS1

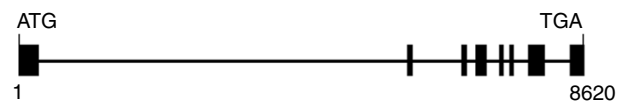

g.7961_7975 delete TCCTTGGTGAAGGTA g.7961_7973 insert ATGTATATATACT
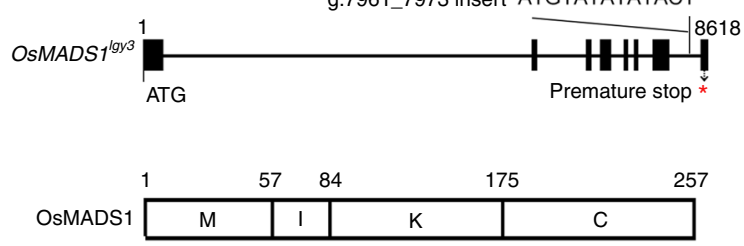

OsMADS1 $^{1 \mathrm{lgy} 3}$\begin{tabular}{l|l|l|l|l|}
\hline $\mathrm{M}$ & \multicolumn{1}{c}{57} & \multicolumn{1}{c}{1} & \multicolumn{1}{c}{175} \\
\hline
\end{tabular} e

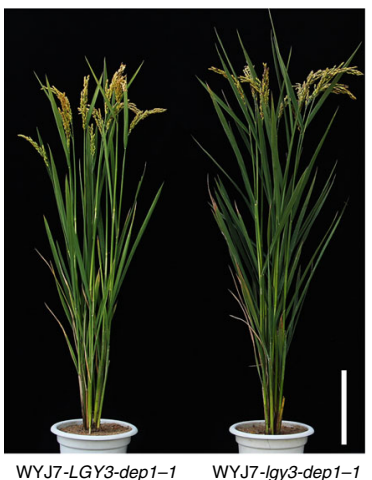

f

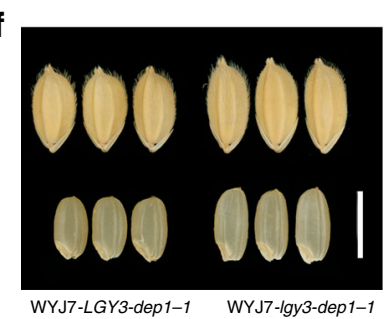

$\mathbf{k}$
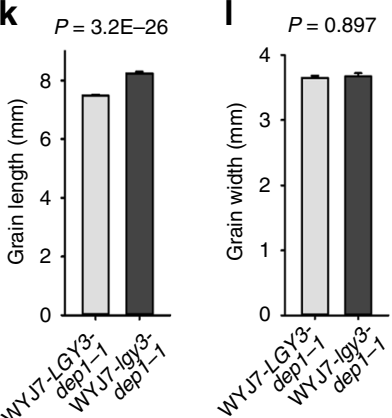

g

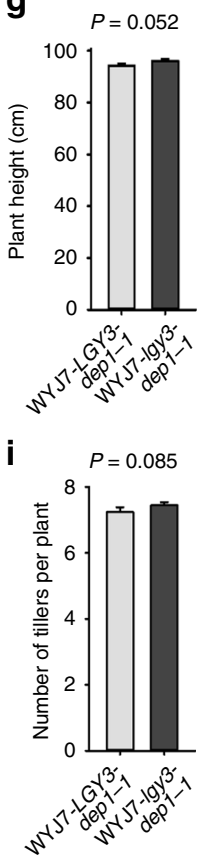

h
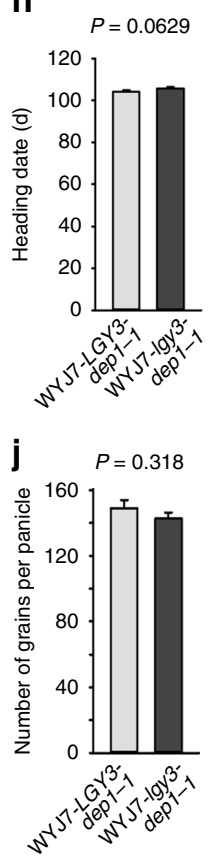

m

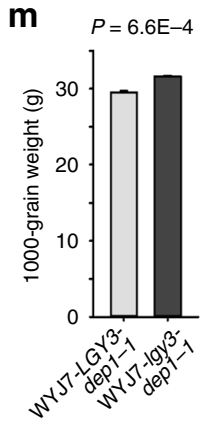

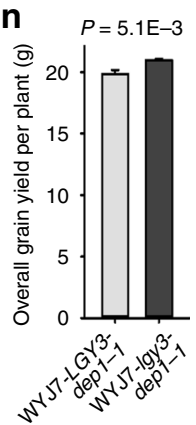

Fig. 1 The interaction between DEP1 and OsMADS1 regulates grain size and yield potential of rice. a Grain morphology. Scale bar: $5 \mathrm{~mm}$. b QTL mapping for grain length and grain yield. c The positional cloning of $q L G Y 3$. The candidate region was mapped to a $\sim 8.9$ Kbp genomic DNA region lying between markers XP22 and XP23. The numbers below the line indicate the number of recombinants recovered between qLGY3 and markers. $\mathbf{d}$ Allelic variations of OsMADS1 between RD23 and L-204. In the schematic illustration of OsMADS1 functional domains, M represents the MADS domain, I represents the intervening domain, $\mathrm{K}$ represents the keratin-like domain, and $\mathrm{C}$ represents the $\mathrm{C}$-terminal domain. e The gross morphology of NIL plants. Scale bar: $15 \mathrm{~cm}$. f Grain size and shape. Scale bar: $5 \mathrm{~mm}$. g-n A field-based comparison of the WYJ7-LGY3-dep1-1 and WYJ7-lgy3-dep1-1 plants: $\mathbf{g}$ Plant height; $\mathbf{h}$ Heading date; $\mathbf{i}$ The number of tillers per plant; $\mathbf{j}$ The number of grains per panicle; $\mathbf{k}$ Grain length; I Grain width; $\mathbf{m}$ 1,000-grain weight; and $\mathbf{n}$ The overall grain yield per plant. All phenotypic data were measured from the paddy-grown NIL plants grown under normal cultivation conditions. Data shown as mean \pm s.e.m. $(n=288)$. Student's $t$-test was used to generate the $P$ values

those produced by transgenic NPB-LGY3 plants in which OsMADS1 had been knocked down by RNAi (Supplementary Fig. $3 g, h)$. There was little difference in the length of grains formed by over 10 independent transgenic rice plants expressing the Nipponbare LGY3 cDNA driven by its native promoter, whereas constitutive expression of the Nipponbare LGY3 cDNA driven by the rice Actin promoter resulted in abnormalities in the lemma and palea development (Supplementary Fig. 3h), a phenotype which was indistinguishable from that formed by Ubi::OsMADS1 plants as described previously ${ }^{32}$. Thus, the product of OsMADS1 $1^{\lg y 3}$ appears to be a dominant negative regulator of OsMADS1 function. 
Combining $\lg y 3$ and $\operatorname{dep} 1-1$ alleles improves quality and yield. Haplotype analysis at the OsMADS1 locus revealed that the L-204 haplotype involving truncating splice-site mutation is common within O. nivara accessions and tropical japonica germplasm, but it does not appear to occur within the elite indica and temperate japonica rice varieties (Supplementary Data 1), indicating that the lgy 3 allele has not been exploited in high-yielding rice breeding programs. The dep1-1 allele on the other hand has been widely used to develop high-yielding rice varieties by Chinese breeders $^{8,10}$, therefore, it was of interest to characterize the effect on grain yield of combining elite alleles at the LGY3 and DEP1 loci. The two NILs plants, WYJ7-lgy3-dep1-1 and WYJ7-LGY3-dep1-1, did not differ from one another with respect to heading date, plant height, tiller numbers per plant, and grain numbers per panicle (Fig. 1e, g-j), but WYJ7-lgy3-dep1-1 plants produced longer and heavier grains than those formed by WYJ7-LGY3dep1-1 plants (Fig. 1f, $\mathrm{k}-\mathrm{m}$ ). Over three successive years of field trialling, WYJ7-lgy3-dep1-1 out-yielded WYJ7-LGY3-dep1-1 by an average of $10.4 \%$ (Fig. 1n). Meanwhile, WYJ7-lgy3-dep1-1 plants produced much better quality in terms of grain length-towidth ratio and grain chalkiness (Fig. 2 and Supplementary Data 2). Thus, pyramiding of the lgy3 and dep1-1 alleles provides a promising strategy for simultaneously improving rice yield and grain quality above what is currently achievable.

DEP1 interacts with MADS-domain transcription factors. In rice, OsMADS1 is one of E-class MADS-box genes involved in the ABCDE model of flower development ${ }^{33,34}$. To uncover the molecular mechanisms underlying genetic interaction between the DEP1 and OSMADS1 genes, we performed a yeast two-hybrid screen to identify DEP1-interacting proteins, and found that both DEP1 and truncated dep1-1 proteins interacted with OsMADS1 (Supplementary Fig. 4a). The DEP1-OsMADS1 interactions in

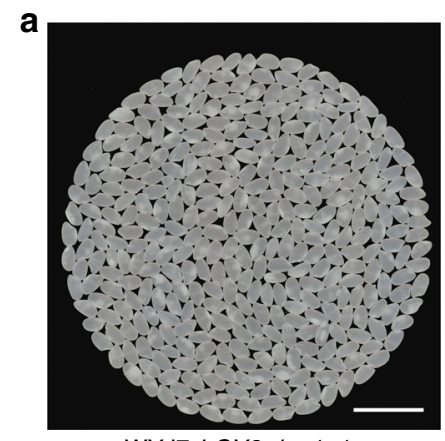

WYJ7-LGY3-dep1-1

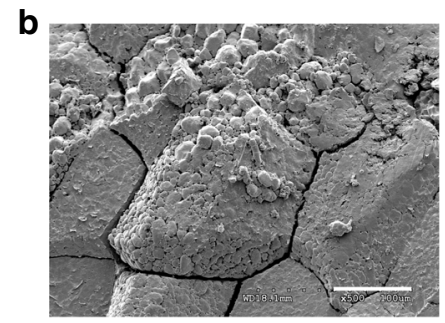

WYJ7-LGY3-dep1-1

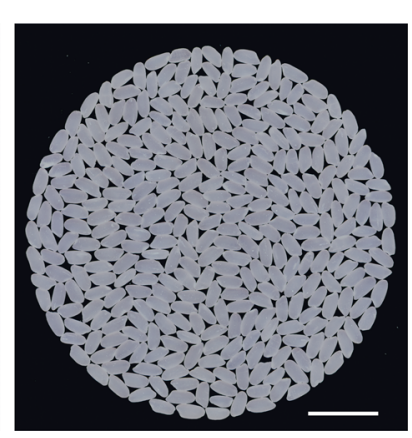

WYJ7-lgy3-dep1-1

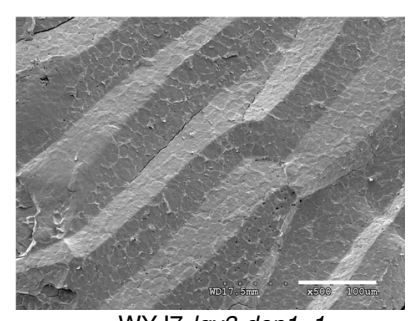

WYJ7-lgy3-dep1-1
Fig. 2 Introduction of the lgy3 allele into the high-yielding variety results in the improvement of rice grain quality. a Comparisons of grain chalkiness and endosperm transparency between the WYJ7-LGY3-dep1-1 and WYJ7lgy3-dep1-1 plants. Scale bar: $15 \mathrm{~mm}$. b Scanning electron microscopy images of the transverse sections of starch granule from the WYJ7-LGY3dep1-1 and WYJ7-Igy3-dep1-1 plants. Scale bar: $50 \mu \mathrm{m}$. The endosperm of the NIL plants carrying the lgy3 allele comprised largely sharp edged, compactly arranged polygonal starch granules planta were confirmed by the split firefly luciferase complementation (SFLC) in tobacco leaf epidermal cells and coimmunoprecipitation assays (Fig. 3a, b). We also found that OsMADS1 ${ }^{1 \operatorname{lgy} 3}$ could interact with both DEP1 and dep1-1 proteins (Fig. 3a), implying that it was unlikely that the $\mathrm{C}$ domain of OsMADS1 was critical for its interaction with DEP1.

We next constructed various domain-based deletions of OsMADS1 for SFLC assays, and found that both I and K domains were necessary and sufficient for the interaction with DEP1 (Fig. 3c). Previous studies have shown that the K domain is required for MADS-domain protein function and is highly conserved across many plant taxa ${ }^{35}$, which is consistent with the findings that DEP1 could interact with other MADS-domain proteins (Supplementary Fig. 4b). In addition, we constructed various domain-based deletions of DEP $1^{10}$, and further SFLC assays demonstrated that the vWFC domain of DEP1 was required for the DEP1-OsMADS1 interaction (Supplementary Fig. 5). The transient transcriptional activity assays showed that $C$ domain-dependent OsMADS1 transactivation activity was substantially enhanced when both OsMADS1 and DEP1 were combined (Fig. 3d). Although transactivation activity of either OsMADS1 $1^{\Delta \mathrm{K}}$ (lacking the $\mathrm{K}$ domain) or OsMADS1 $1^{\Delta \mathrm{I}+\Delta \mathrm{K}}$ (lacking both $\mathrm{I}$ and $\mathrm{K}$ domains) was stronger than that of OsMADS1, its activity was not promoted, but was retained when OsMADS1 $^{\Delta \mathrm{K}}$ (or OsMADS1 ${ }^{\Delta \mathrm{I}+\Delta \mathrm{K}}$ ) and DEP1 were combined (Fig. 3d). These results suggest that DEP1 physically interacts with transcription factor OsMADS1, and the DEP1-OsMADS1 interaction promotes the transactivation activity of OsMADS1.

OsMADS1 and DEP1 co-regulate common target genes. The constitutive knockdown of OsMADS1 in both WYJ7-DEP1 and WYJ7-dep1-1 plants resulted in a phenotype similar to that of osmads1 mutant ${ }^{29,31}$, which produced an elongated palea and lemma (Supplementary Fig. 6). These genetic results suggest that $D E P 1$-mediated regulation of grain size and shape is dependent upon OsMADS1 function. To identify common target genes of the DEP1-OsMADS1 regulatory module, we next compared the genome-wide transcriptional profiles in the developing panicles and spikelet hulls of the NILs WYJ7-LGY3-DEP1, WYJ7-LGY3dep1-1, WYJ7-lgy3-DEP1, and WYJ7-lgy3-dep1-1 plants using RNA-seq. Comparisons of RNA-seq and ChIP-seq data ${ }^{36}$ revealed a total of 451 genes, which were cooperatively regulated by the DEP1-OsMADS1 regulatory module (Fig. 4a and Supplementary Data 3). Among these were certain AP2/ERF and MADS-box family members, along with genes encoding proteins involved in the biosynthesis of, transport of, and response to auxin (Supplementary Data 3). Quantitative RT-PCR assays confirmed that the GARP family genes OsKANADI2 and OsKANADI4, the MADS-box family genes OsMADS34 and OsMADS55, the auxin efflux carrier OsPIN1a, and the auxin response factor genes OsARF9 and OsARF14 were all more strongly transcribed in WYJ7-LGY3-dep1-1 plants than in either WYJ7-LGY3-DEP1 or WYJ7-lgy3-dep1-1 plants (Fig. 4b). These results indicate that dep1-1 and OsMADS1 cooperatively regulate a common set of target genes during development of spikelets in rice. Further ChIP-PCR and EMSA assays revealed that both OsMADS1 and OsMADS1 1 lgy3 were able to bind the promoter regions of OsMADS55, OsKANADI4, OsPIN1a, and OsARF9, and that the DNA-binding affinity was unaffected by variations in the C domain sequence (Fig. 4c, d).

Moreover, ChIP-PCR assays demonstrated that the in vivo association of OsMADS1 with a same promoter region of target genes examined was unaffected by the presence or absence of dep1-1 (Fig. 4e), indicating that the effect of the DEP1-OsMADS1 interaction on target gene expression was not 

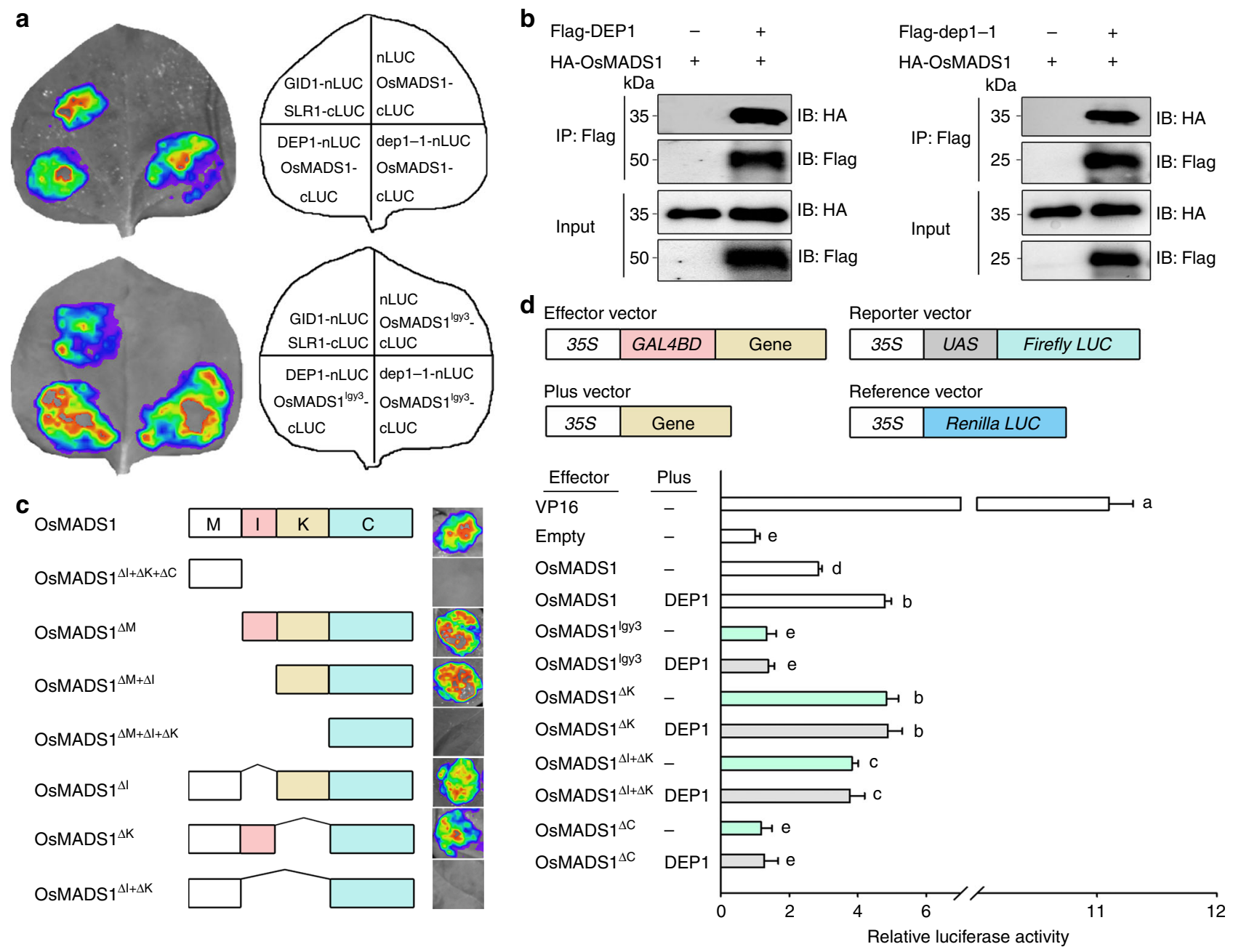

Fig. 3 The physical interaction between DEP1 and OsMADS1. a SFLC assays. nLUC-tagged DEP1 (or dep1-1) was co-transformed into tobacco leaves along with either the CLUC-targeted OsMADS1 or CLUC-targeted OsMADS1gy3. The interaction of the rice GA receptor GID1 and the rice DELLA protein SLR1 were used as positive control. b Co-immunoprecipitation assays of Flag-DEP1 and HA-OsMADS1, and Flag-dep1-1 and HA-OsMADS1. IB Immunoblot, IP immunoprecipitation. $\mathbf{c}$ The image shows a SFLC assay in which nLUC-tagged deleted and non-deleted versions of OsMADS1 were co-transformed into tobacco leaves along with cLUC-DEP1. d The effect of the OsMADS1-DEP1 interaction on OsMADS1-induced transactivation activity. Deleted and nondeleted versions of OSMADS1 were fused to the GAL4-binding domain (GAL4BD). The relative activity of firefly luciferase (LUC) under control of the GAL4-binding element UAS was measured. Renilla luciferase (REN) activity was used as reference and VP16 as the positive control. Data shown as mean \pm s.e.m. $(n=6)$. Statistical analyses were performed by Duncan's multiple range tests. The presence of the same lowercase letter denotes a non-significant difference between means $(P>0.05)$

triggered by the resulting changes in the DNA-binding affinity of OsMADS1. Rice OsARF9 is homologous to A. thaliana ARF2 and S. lycopersicum SIARF9, which negatively control, respectively, seed size in $A$. thaliana ${ }^{37}$ and fruit weight in tomato ${ }^{38}$. Transient expression assays in rice protoplasts showed that LUC activity driven by the OSARF9 promoter was moderately induced by OsMADS1 on its own, but was substantially enhanced when both OsMADS1 and DEP1 were combined (Fig. 4f), which is well consistent with the experimental results that OsMADS1 and DEP1 acted as the repressors in regulating grain size (Fig. 1a, f). Furthermore, LUC activity was moderately induced by OsMADS1 $^{\Delta \mathrm{K}}$ on its own, but not enhanced when both DEP1 and OsMADS1 ${ }^{\Delta \mathrm{I}+\Delta \mathrm{K}}$ were combined (Fig. 4f). In addition, OsMADS1-induced LUC activities were promoted when assays were performed in the presence of either DEP1 or dep1-1, whereas mutations in the $\mathrm{C}$ domain of OsMADS1 abolished the DEP1-promoted transactivation activity (Fig. 4g). Taken together, these results indicate that DEP1 acts as a functional OsMADS1 cofactor in controlling grain size through regulation of the common target genes.

OsMADS1 is a key downstream effector of G-protein $\beta \gamma$ dimers. Heterotrimeric GTP-binding proteins (G proteins) are signal transduction components that mediate multiple intracellular responses to external stimuli in diverse eukaryotic organisms. The rice genome encodes one $\mathrm{G} \alpha$ subunit (RGA1), one $\mathrm{G} \beta$ subunit (RGB1), two canonical G $\gamma$ subunits (RGG1 and RGG2), and three non-canonical G $\gamma$ subunits (GS3, DEP1, and OsGGC2) 39. It is well-known that, following dissociation of $G \alpha$ subunit, $\mathrm{G} \beta \gamma$ dimers act as a functional unit to activate a large number of its own effectors ${ }^{40}$. Previous studies have shown that RGB1, RGG1, and RGG2 were datable in the plasma membrane ${ }^{41}$. However, recent studies suggested potential nuclear localization or translocation of the G $\beta \gamma$ dimer in mammalian cells, which co-localized with the AP-1 transcription factor and recruited 
histone deacetylases to inhibit AP-1 transcriptional activity ${ }^{42,43}$. To investigate whether transcription factor OsMADS1 acts as a downstream effector of $G \beta \gamma$ subunits in rice, we performed the analysis of protein-protein interactions in planta. Although the canonical G-protein $\gamma$ subunits RGG1 and RGG2 do not contain vWFC domain, SFLC assays showed that all of the rice G $\gamma$ subunits interacted with OsMADS1 (Fig. 5a). In addition, the rice G $\beta$ subunit RGB1, but not the rice $\mathrm{G} \alpha$ subunit RGA1, was associated a
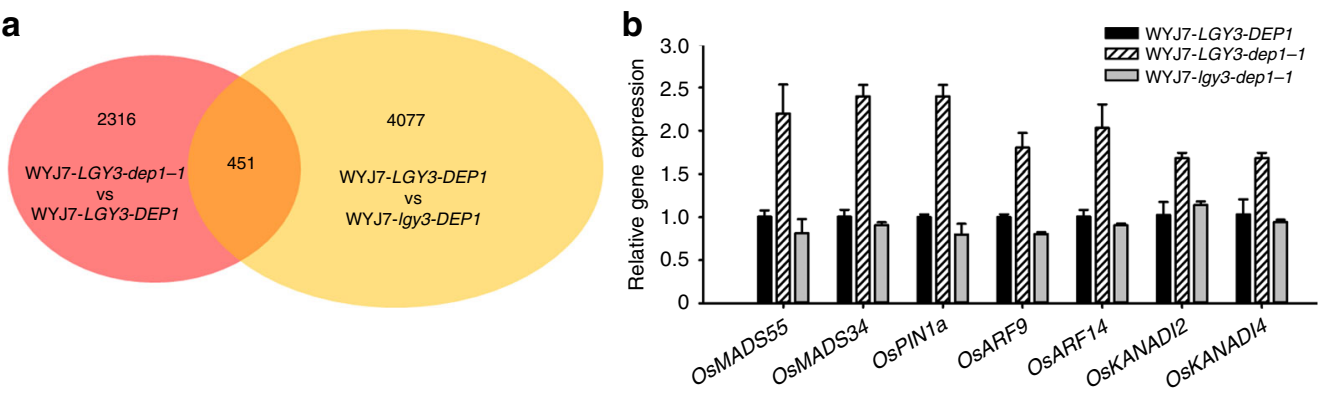

C
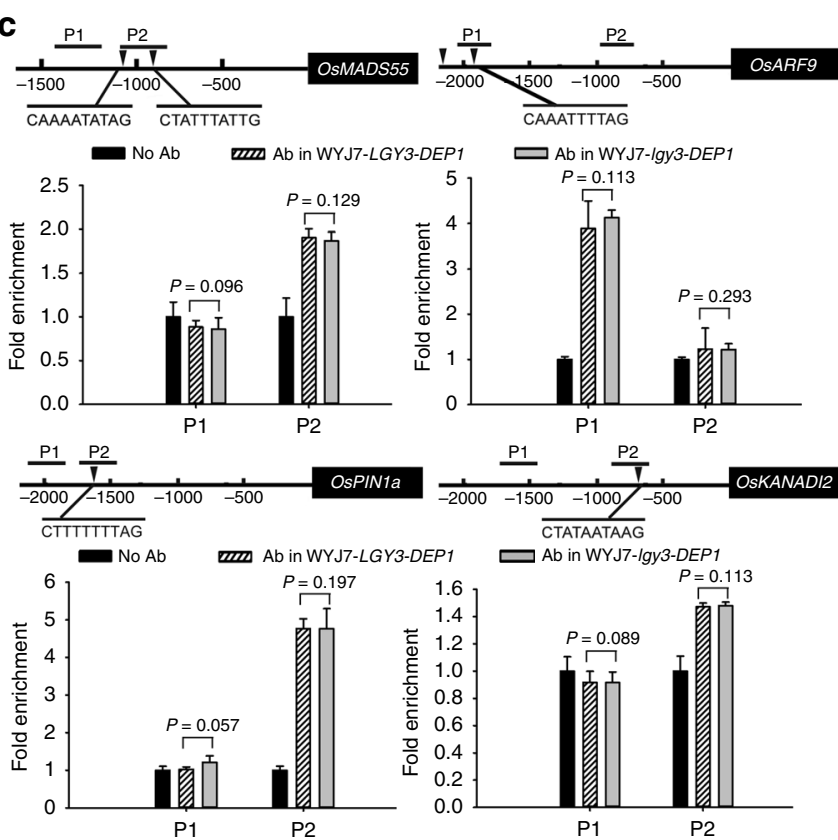

f
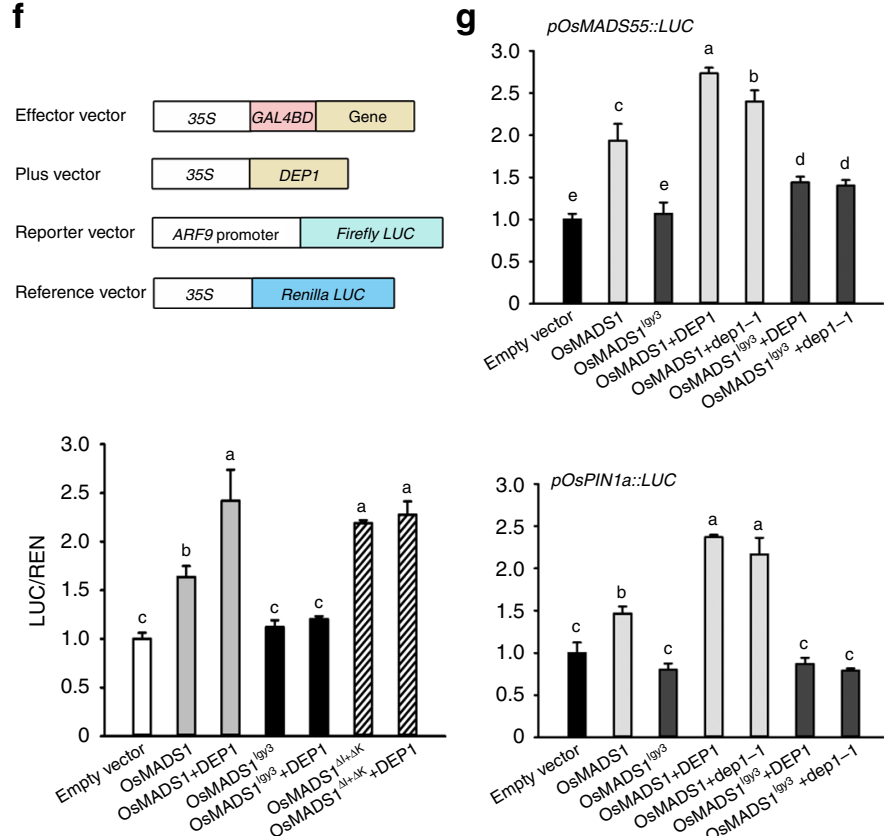

d OSMADS1 $19 \mathrm{gy}$-GST -

Biotin probe Cold probe
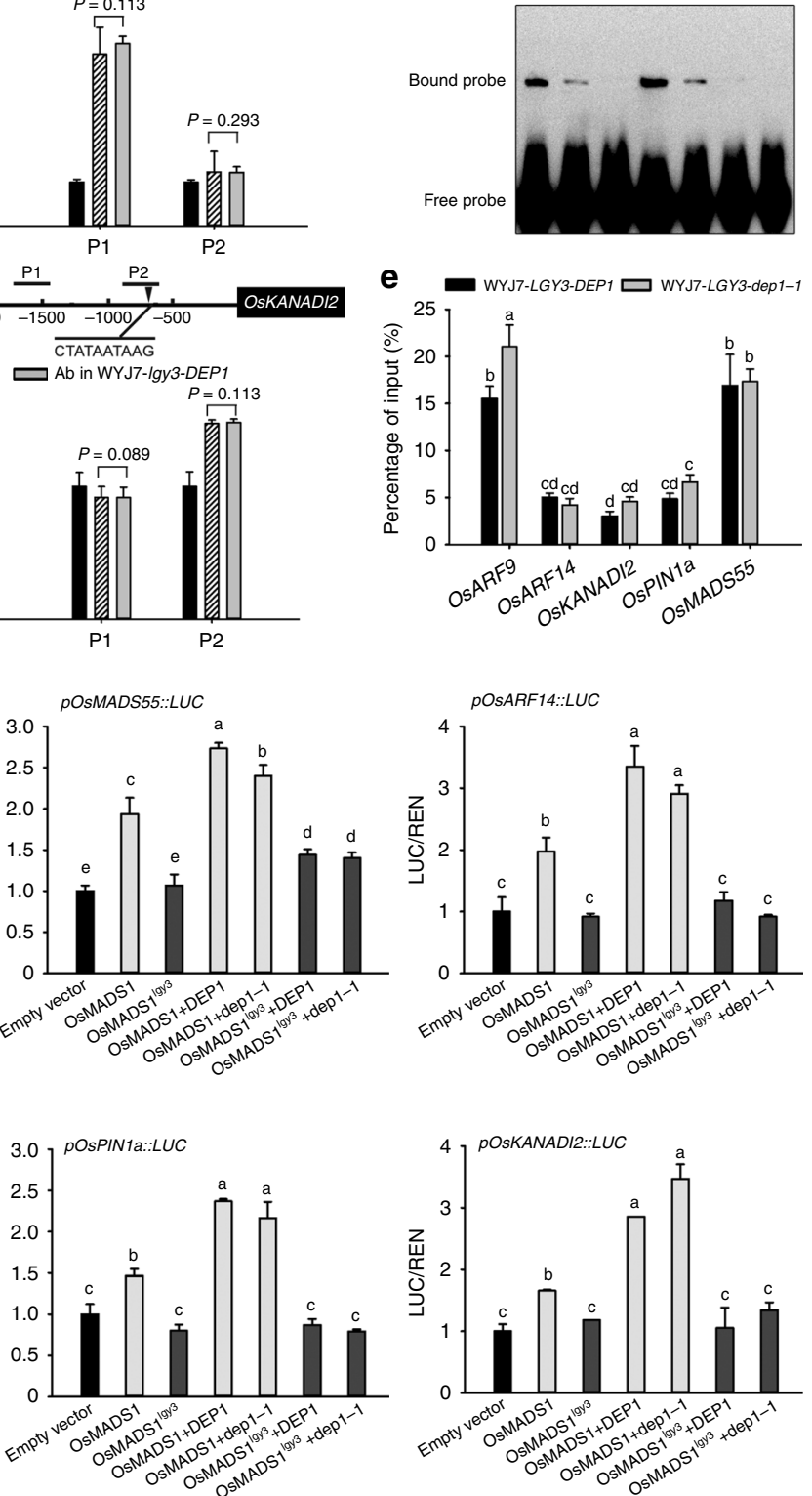

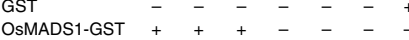


with OsMADS1 (Fig. 5a). Interestingly, the nuclear-localized RGB1-GFP fusion proteins were datable in both the plasma membrane and the nucleus of root cells in transgenic rice plants expressing RGB1-GFP under the control either of its native promoter (Supplementary Fig. $7 \mathrm{a}$ ) or of the cauliflower mosaic virus (CaMV) $35 \mathrm{~S}$ promoter ${ }^{10}$. Further transient expression assays showed that dep1-1-GFP and GS3-GFP fusion proteins were datable in both the plasma membrane and the nucleus (Supplementary Fig. 7b). To investigate whether the nuclearlocalized dep1-1 protein was involved in controlling plant height and grain size, we generated transgenic rice plants carrying either pActin::HA-NLS-dep1-1 or pDEP1::HA-dep1-1 construct. We found that the nuclear-localized HA-NLS-dep1-1 fusion protein was associated with the formation of a semi-dwarf phenotype (Supplementary Fig. 7c), which was similar to that of the transgenic rice plants expressing dep1-1 under control of either the constitutive promoter ${ }^{10}$ or its native promoter (Supplementary Fig. 7c). These results suggest that the nuclear translocation of DEP1 is involved in G-protein signaling in rice.

The transgenic rice plants carrying the pActin::GS3-GFP construct also displayed a semi-dwarf phenotype with the reduced size of grains (Supplementary Fig. 7d), the phenotypes that were indistinguishable from that of the transgenic plants carrying the $p D E P 1::$ dep 1-1 construct ${ }^{10}$. We also showed that the GS3-GFP fusion protein was detectable in the plasma membrane and the nucleus of rice root cells (Supplementary Fig. 7e, f). In addition, the transgenic rice plants overexpressing either RGB1, $R G G 1$, or $R G G 2$ formed grains that were much smaller than those formed by non-transgenic control plants, a phenotype reminiscent of that of dep1-1 overexpressor ${ }^{10}$ (Fig. 5b). In addition, introduction of the $\lg 33$ allele into the transgenic rice plants overexpressing either $R G B 1$ or $H A-R G G 1$ substantially enhanced grain length and formed more slender grains (Fig. 5b). Further protoplast transient expression assays showed that OsMADS1induced LUC activities of the common target genes examined were substantially enhanced when GS3 and OsMADS1 were combined (Fig. 5c), representing a response similar to that regulated by the DEP1-OsMADS1 interaction (Fig. 4g). Taken together, these results reveal that both DEP1 and GS3 appear to be cofactors of OsMADS1 in controlling the grain size through regulation of common target genes.

Combining OsMADS1 ${ }^{\lg y 3}$ with $g s 3$ enhances yield and quality. The gs 3 allele has been used widely in indica rice breeding programs $^{5,15}$. The effect of allelic combinations on grain yield and quality was explored by generating NILs carrying allelic combinations of $q G S 3$ and $q L G Y 3$ loci in the indica variety $\mathrm{RD} 23$ (hereafter RD23-LGY3-gs3) (Fig. 5d, e). The two NILs RD23LGY3-gs3 and RD23-lgy3-gs3 did not differ from one another with respect to either heading date, plant height, or tiller number (Fig. 5f-h), although the number of grains per panicle was slightly reduced (Fig. 5i). Both RD23-LGY3-gs3 and RD23-lgy3-GS3 plants formed longer grains than those formed by RD23-LGY3GS3 plants (Fig. 5e), while RD23-lgy3-gs3 plants formed substantially longer and heavier grains than those formed by either RD23-LGY3-gs3 or RD23-lgy3-GS3 plants (Fig. 5e, j, k). Over three successive years of field trials, RD23-lgy3-gs3 proved to be on average $\sim 10.9 \%$ more productive than RD23-LGY3-gs3 (Fig. 51). In addition, the introduction of the $\lg y 3$ allele into elite variety $\mathrm{RD} 23$ also improved grain appearance quality (Supplementary Figs. 8a, 9a and Supplementary Data 2).

Because the mode of inheritance of the OsMADS1 loy3 mutation was semi-dominant (Supplementary Fig. 1), we next investigated whether the lgy 3 allele was able to simultaneously improve grain quality and yield in indica hybrid rice. The two-line hybrid combinations ${ }^{44}$ were developed by crossing a photothermosensitive genic male sterile PA64S either with a restorer line 9311 (which carries the LGY3 and $g s 3$ alleles, hereafter 9311LGY3-gs3) or with a 9311 NIL plants (which carries the $\lg y 3$ and gs3 alleles, hereafter 9311-lgy3-gs3). The effects of the $\lg y 3$ allele were to enhance both grain length and weight (Fig. $5 \mathrm{~m}-\mathrm{o}$ ), to increase grain yield by a mean of $7.1 \%$ (Fig. 4p), and to substantially improve the grain quality (Supplementary Figs. 8b, $9 \mathrm{~b}$ and Supplementary Data 2). Thus, combining gs3 with $\lg y 3$ may be an efficient method for breeding higher-quality and higher-yielding rice above what is currently achievable.

\section{Discussion}

Heterotrimeric G-protein signaling is an evolutionarily conserved mechanism found in all eukaryotes. In humans, the heterotrimeric G-protein complexes comprising $23 \mathrm{G} \alpha, 5 \mathrm{G} \beta$, and $12 \mathrm{G} \gamma$ subunits are important signal transducers that mediate intracellular responses to a broad range of external stimuli. $G \alpha$ subunit is a molecular switch for bimodal activities with a guanosine diphosphate (GDP)-bound "off" mode and a GTP-bound "on" mode $^{39}$. In the absence of ligands, G $\alpha$ subunit binds to GDP and forms an inactive heterotrimer with $\mathrm{G} \beta \gamma$ dimer. In the presence of ligands, G protein-coupled receptors (GPCRs) are activated and then undergo a conformational change, which in turn promote the exchange of GDP for GTP on G $\alpha$ subunit, thereby triggering specific downstream effectors of both activated $G \alpha$ and $G \beta \gamma$ subunits ${ }^{39}$. In plants, the G-protein complex is also composed of $\mathrm{G} \alpha, \mathrm{G} \beta$, and $\mathrm{G} \gamma$ subunits, integrating multiple environmental and hormonal signals, and plays the important roles in the regulation

\footnotetext{
Fig. 4 DEP1 and OsMADS1 cooperatively regulate a common set of target genes. a The number and overlap of DEP1 and OsMADS1 targets. RNA-seq experiments were performed based on template extracted from young NIL panicles. $\mathbf{b}$ Transcript abundances of the common target genes examined in the indicated NILs plants. The relative abundances in NIL-LGY3-DEP1 plants was set to be one. Data shown as mean \pm s.e.m. $(n=6)$. c ChIP assays of the WYJ7-LGY3-DEP1 and WYJ7-Igy3-DEP1 plants. The DNA fragments either containing or non-containing CArG-box motifs (arrowed) in the promoter regions of the targets OsMADS55, OsKANADI42, OsPIN1a, and OsARF9 were used. Data shown as mean \pm s.e.m. $(n=6)$. A Student's $t$-test was used to generate the $P$ values. d EMSA assays. Competition for either OsMADS1-GST or OsMADS1 1 gy3 - GST binding was performed with $10 \times$ and $50 \times$ unlabeled probes containing the CArG-box motifs, respectively. e ChIP analysis of the OsMADS1 interaction with the promoter regions of OsMADS55, OsKANADI2, OsPIN1a, OsARF9, or OsARF14 with amplicon sequences shown in Fig. 3c. Data shown as mean \pm s.e.m. $(n=3)$. Statistical analyses were performed by Duncan's multiple range tests. $\mathbf{f}$ The effect of deleting the $\mathrm{K}$ domain on the DEP1-dependent promotion of OsMADS1-induced transactivation activity of the OSARF9 promoter. The ratio of LUC to REN activity was monitored in rice protoplasts co-transfected with various effector and reporter constructs. The LUC/REN activity obtained from a co-transfection with an empty effector construct and reporter construct was set to be one. Data shown as mean \pm s.e.m. $(n=6) . \mathbf{g}$ The DEP1-OsMADS1 interaction promotes the OSMADS1-induced target gene transactivation activity. The LUC/REN activity obtained from a cotransfection with an empty effector construct and indicated reporter constructs was set to be one. Data shown as mean \pm s.e.m. $(n=6)$. Statistical analyses were performed by Duncan's multiple range tests. The presence of the same lowercase letter denotes a non-significant difference between means $(P>0.05)$
} 
of plant growth, metabolism, and adaption in relation to biotic and abiotic stress conditions ${ }^{10,45-50}$.

In humans, the functional specificity of G-protein signaling is possible through over 1,380 unique combinations of heterotrimeric $\mathrm{G}$ proteins ${ }^{39}$. Conversely, most plants have only one $G \alpha$, one $G \beta$, and several $G \gamma$ subunits ${ }^{51}$; hence, rising questions regarding how the simple heterotrimer variants controls the various intercellular processes in plants. Unlike human GPCR- a
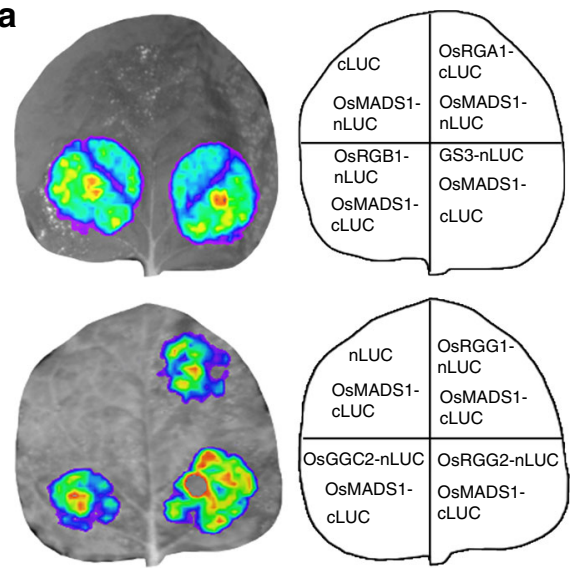

C
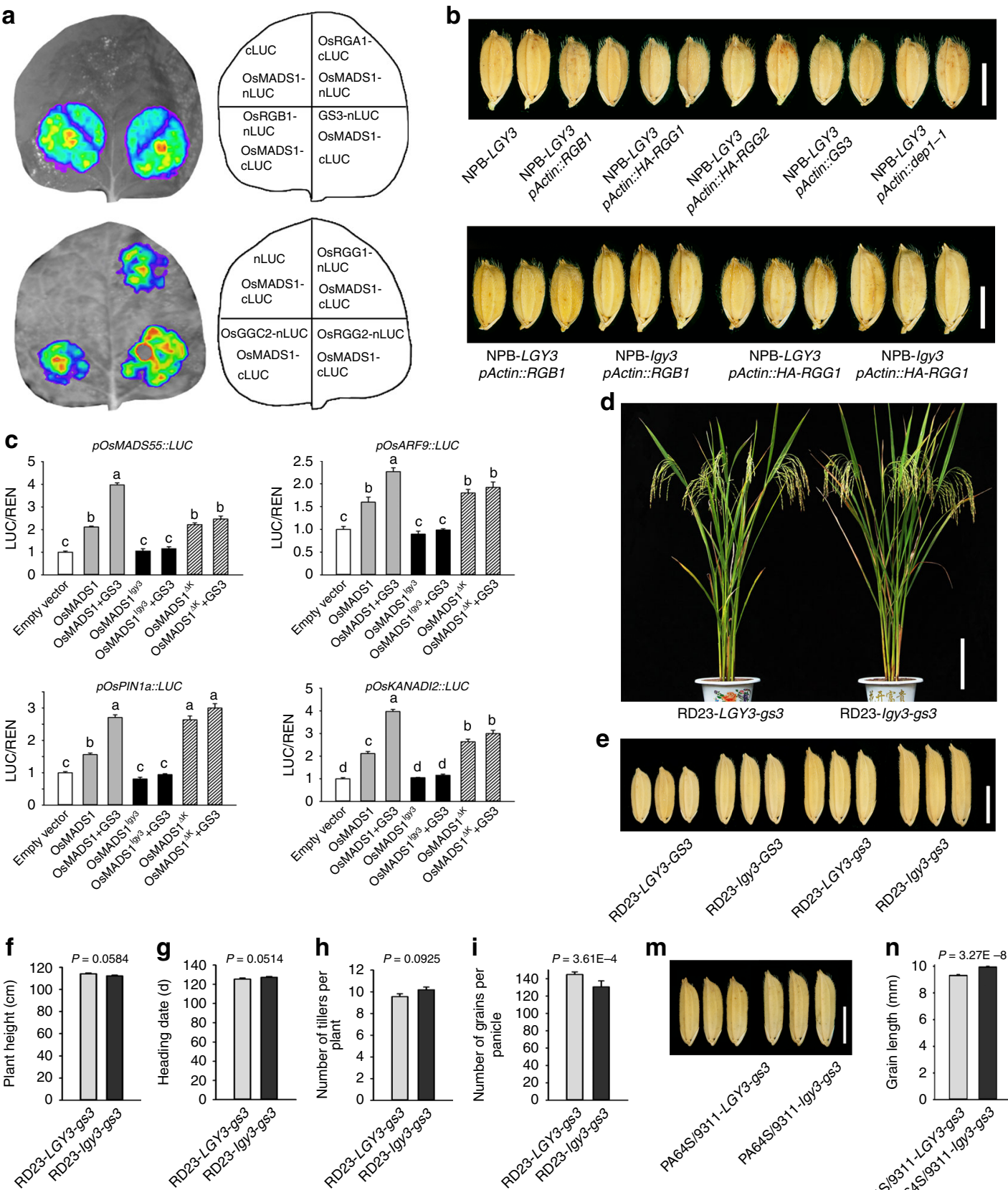

e

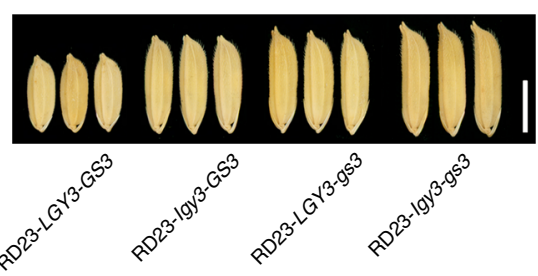

m
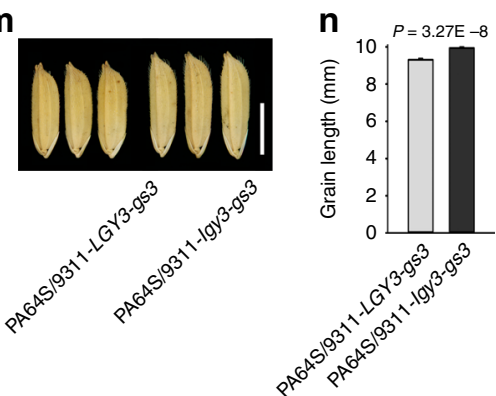
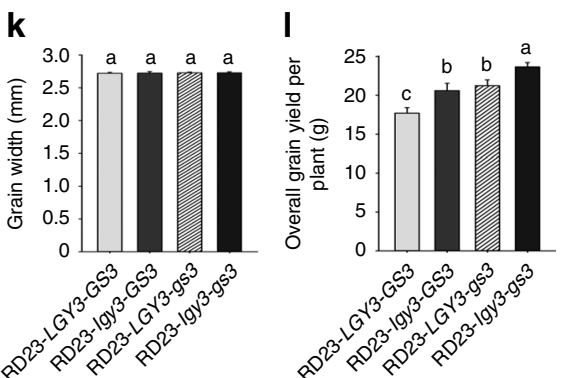
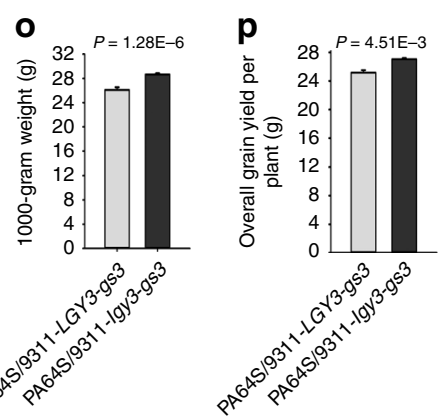
dependent G-protein activation-deactivation cycle, the plant $G \alpha$ subunit can be self-activation, it was proposed that the acceleration of GTP hydrolysis maybe more important for G-protein activation-deactivation cycle ${ }^{39}$. Recent studies showed that Nod factor receptors (NFR1/NFR5) interacted with soybean $\mathrm{G} \alpha$ and RGS proteins, which in turn promoted the formation of the inactive heterotrimer, thereby regulating the nodule formation and development ${ }^{52}$. It has been shown that maize FEA2 (FASCIATED EAR2) protein, an Arabidopsis CLV2 homolog, interacted with maize $\mathrm{G} \alpha$ subunit CT2 (COMPACT PLANT2), resulting in the control of stem cell homeostasis in shoot apical meristems ${ }^{53}$. These results revealed that the plant heterotrimeric G-protein signaling specificity is possible through the formation of multiple complexes between $G$ proteins and receptor-like kinases, and these interacting partners may be essential to sense ligands and distinguish extracellular stimuli, and may also transduce the signals into the cell and finally induce intercellular responses.

Following stimulation of GPCRs at the cell surface, the activated $\mathrm{G} \alpha$ and detached $\mathrm{G} \beta \gamma$ dimers classically act as two functional modules ${ }^{40}$, and consequently trigger specific downstream effectors and intercellular responses. However, the signaling mechanisms downstream of the heterotrimeric $G$ proteinmediated intercellular responses remain poorly understood. The role of $G \beta \gamma$ dimers was initially proposed to be prevention of spontaneous activation of the $\mathrm{G} \alpha$ subunit, but systematic screens for the protein-protein interactions with $G \beta$ and $G \gamma$ subunits identified several interacting partners that were localized at different sub-cellular sites distinct from the plasma membrane ${ }^{42,43}$. Recent studies demonstrated that G $\beta \gamma$ dimer was able to colocalize with transcription factor AP-1 in the nucleus, and recruited histone deacetylases to inhibit activity of AP-1 transcription $^{42,43}$. Here, we showed that the GS3-GFP fusion protein was able to be detected in the nucleus of protoplasts isolated form leaf sheath of 10-day-old seedlings of the transgenic rice plants (Supplementary Fig. 7), consistent with the pervious observations that both RGB1-GFP and dep1-1-GFP fusion proteins were detectable in the nucleus ${ }^{10}$. The outcome suggests potential nuclear localization or translocation of the G $\beta \gamma$ dimers found in both animals and plants.

In this study, we identified a rice quantitative trait locus $L G Y 3$, which is synonymous with OsMADS1, a gene encoding a MADSdomain transcription factor ${ }^{29}$. In the further experiments, we demonstrated that the lgy3 allele is a dominant negative mutation causing an alternatively spliced protein OsMADS1 1 gy 3 , in which the terminal 37 residues were truncated and an additional 5 residues were added to its predicted $\mathrm{C}$ domain of OsMADS1 (Fig. 1d). The effect of the lgy3 allele is to promote cell proliferation in longitudinal direction during the spikelet development in rice (Supplementary Fig. $3 b-d$ ), resulting in the formation of a more slender grain and better appearance quality, which also applies in combing with the high-yielding dep1-1 allele (Fig. 1f, Supplementary Data 2). Indeed, Both RNA-seq and ChIP-seq analysis demonstrated that OsMADS1 and DEP1 share common target genes, such as the genes involved in auxin biosynthetic pathway and the auxin-signaling related genes (Fig. 4a, b and Supplementary Data 3), which is in accordance with the function of dep1-1 in regulating the activity of shoot apical meristems and panicle branching 8,10 . More importantly, we have found that DEP1, as well as truncated dep1-1, interacted directly with OsMADS1 (Fig. 3a, b). Both DEP1 and dep1-1 are positive regulators of OsMADS1-mediated transcription, but this interaction is sufficient, but not necessary for enhancement of the transcriptional activity of OsMADS1 (Fig. 4f, g). SFLC assays also showed that rice G $\beta$ subunit RGB1 could interact directly with OsMADS1 (Fig. 5a), which is consistent with the note that potential nuclear localization or translocation of animal $G \beta \gamma$ dimers $^{42,43}$. Our findings suggest that rice $G \beta \gamma$ dimers can be colocalized (or translocated) into the nucleus and interacted directly with MADS-domain transcription factors (i.e., OsMADS1), thereby regulating downstream target gene expression.

It is known that all set of the MADS-domain proteins contain a DNA-binding domain named MADS domain ( $\mathrm{M}$ domain) that is located at the $\mathrm{N}$ terminal ${ }^{54}$. MIKC-type (also known as type II) MADS-domain proteins contain three additional domains, including an Intervening domain (I domain), Keratin-like domain ( $\mathrm{K}$ domain), and $\mathrm{C}$-terminal domain ( $\mathrm{C}$ domain). The $\mathrm{K}$ domain, as the most highly conserved and characteristic one, together with I domain are responsible for protein interaction and dimerization $^{55}$. Our results showed that both I domain and $\mathrm{K}$ domain were indispensable for the interaction with the $\mathrm{G} \gamma$ subunit DEP1 (Fig. 3c). The transcriptional activities of OsMADS1 were enhanced when OsMADS1 was combined with either DEP1 or dep1-1 (Fig. 3d), suggesting that G $\beta \gamma$ subunits interact with MIKC-type MADS proteins and led to the formation of multiple proteins complexes and consequent increases in transcription activity. The MADS-domain transcription factors have been shown to play the important roles in the control of flowering time, floral organ identity, and growth in response to changes in the external environments (e.g., temperature) ${ }^{56,57}$. Given the evolutionary conservation of both $G$ proteins and MADS-box gene families, our discovery that the interaction between $G \beta \gamma$ subunits and MADS-domain proteins adds a further dimension to current knowledge about cross-talk between the heterotrimeric G-protein signaling and MADS-domain proteins in the regulation of plant growth and development in response to environmental changes.

It has long been believed that the plants that produce more seeds would produce smaller seeds and the plants that produce larger seeds would produce fewer due to genetic trade-offs between these two traits, which are controlled by QTL and influenced by multiple environmental changes. Previous

Fig. 5 The $G \beta \gamma$ dimer is a functional OsMADS1 cofactor in regulating grain size. a SFLC assays. nLUC-tagged either RGA1, RGB1, RGG1, RGG2, GS3, or OsGGC2 was co-transformed into tobacco leaves along with cLUC-targeted OsMADS1. b Grain size and shape. Scale bar: $5 \mathrm{~mm}$. c Effect of the GS3-OsMADS1 interaction on OsMADS1-induced transactivation activity. The LUC/REN activity obtained from a co-transfection with an empty effector construct and indicated reporter constructs was set to be one. Data shown as mean \pm s.e.m. $(n=6)$. Statistical analyses were performed by Duncan's multiple range tests. d-I A field-based comparison of RD23-LGY3-gs3 and RD23-lgy3-gs3 plants: d Plant morphology. Scale bar: $20 \mathrm{~cm}$; e Grain size and shape. Scale bar: $5 \mathrm{~mm}$; $\mathbf{f}$ Plant height; $\mathbf{g}$ Heading date; $\mathbf{h}$ The number of tillers per plant; $\mathbf{i}$ The number of grains per panicle; $\mathbf{j}$ Grain length; $\mathbf{k}$ Grain width; and $\mathbf{I}$ The overall grain yield per plant. A Student's $t$-test was used to generate the $P$ values $(\mathbf{f}-\mathbf{i})$, and Duncan's multiple range tests were performed to generate the $P$ values (j-k). $\mathbf{m}-\mathbf{p}$ A field trial of indica hybrid rice: $\mathbf{m}$ Grain size and shape. Scale bar: 5 mm; $\mathbf{n}$ Grain length; $\mathbf{0} 1,000$-grain weight; and $\mathbf{p}$ The overall grain yield per plant. All phenotypic data were measured from the paddy-grown plants under normal cultivation conditions. Data shown as mean \pm s.e.m. $(n=288)$. Student's $t$-test was used to generate the $P$ values. The presence of the same lowercase letter denotes a non-significant difference between means $(P>0.05)$ 
studies have shown that the loss-of-function mutation of the GS3 gene was associated with the formation of much larger grains ${ }^{15}$, whereas the dep1-1 allele was correlated with an increased grain numbers ${ }^{8,10}$. However, the molecular mechanisms of the two G $\gamma$ subunits-mediated improvement of grain size and grain number remain unclear. In this study, we have demonstrated that both GS3 and DEP1 interact directly with transcription factor OsMADS1, and function as cofactors to promote transcriptional activity of OsMADS1, and cooperatively regulate a common set of target genes, which in turn determine grain size and shape. Our findings of the interaction between G $\beta \gamma$ subunits and MADS-domain transcription factors reveal a new molecular framework for coupling floral organ identity and growth with plasma-membrane-associated G-protein complexes, which links intercellular responses and environmental changes.

Haplotype analysis of the OsMADS1 locus revealed that the lgy 3 allele involving truncating splice-site mutation is common within $O$. nivara accessions and tropical japonica germplasm, but it does not appear to occur within the elite indica and temperate japonica rice varieties (Supplementary Data 1). This result indicates that the $\lg 33$ allele has not been used to breed elite indica and temperate japonica varieties. We have shown that the dep1-1 allele has been widely used in high-yielding japonica rice breeding programs in China ${ }^{8,10}$. Therefore, we employed QTL pyramiding based on combinations of the $\lg y 3$ and dep1-1 alleles with molecular marker-assisted selection. Introduction of the lgy 3 allele into the high-yielding japonica variety WYJ7 carrying the dep 1-1 allele resulted in a 10.4\% advantage in grain yield. More importantly, it also was associated with much better quality in terms of grain length-to-width ratio and grain chalkiness (Fig. 2, Supplementary Figs. 8, 9 and Supplementary Data 2). On the other hand, the gs3 allele has also been widely used in indica hybrid rice breeding programs $s^{15,17}$. In the Liangyoupeijiu ${ }^{44}$ (PA64S x 9311, a high-yielding $\mathrm{F}_{1}$ hybrid rice cultivated in Yangtze River area in China) genetic background, we found that pyramiding of $\lg y 3$ and $g s 3$ alleles enhanced overall grain yield (by about 7\%), with improved grain quality (Supplementary Figs. 8, 9 and Supplementary Data 2). Thus, combining lgy3 allele with high-yield potential-associated dep1-1 and $g s 3$ alleles provides a new strategy in breeding simultaneously for higher grain yield and better grain quality in rice above what is currently achievable.

\begin{abstract}
Methods
Plant materials and growing conditions. A set of 250 RILs population was bred from the cross between L-204 and WYJ7. Details of the germplasm used for the positional cloning and haplotype analysis have been described elsewhere ${ }^{71,18}$. The NILs plants carrying contrasting combinations of the $q L G Y 3$ and $q D E P 1$ loci, $q L G Y 3, q G S 3$ loci were bred by crossing RIL186 six times with the recurrent parents Nipponbare, WYJ7, RD23, and 9311, respectively. Field-grown NIL plants were raised under standard paddy conditions at two experimental stations, one located in Lingshui (Hainan Province) and the other in Hefei (Anhui Province).
\end{abstract}

The positional cloning of qlgy3. Fine-scale mapping of qlgy 3 was based on 4,247 $\mathrm{BC}_{2} \mathrm{~F}_{2}$ populations and $653 \mathrm{BC}_{3} \mathrm{~F}_{2}$ populations derived from the backcross between a selected RIL186 and an indica rice variety RD23 (RD23 as the recurrent parent). The primer sequences used for map-based cloning and genotyping assays were given in Supplementary Data 4.

Transgene constructs. The $O s M A D S 1^{l g y} 3$ coding sequence was amplified from $\mathrm{cv}$. L-204, the OsMADS1 coding sequence and its promoter regions lying $1.9 \mathrm{Kbp}$ upstream of the transcription start site and $3^{\prime}$-UTR lying $1.5 \mathrm{Kbp}$ downstream of the termination site were amplified from cv. Nipponbare, and then inserted into the pCAMBIA1300 (CAMBIA) to generate the POsMADS1::OsMADS1 and pOsMADS1::OsMADS1gy3 expression cassettes. A $317 \mathrm{bp}$ cDNA fragment of OsMADS1 was amplified and used to construct both the pActin::RNAi-OsMADS1 and $p O s M A D S 1:: R N A i-O s M A D S 1$ transgenes as described elsewhere ${ }^{3}$. To generate the p35S::OsMADS1 lgy3-GFP and p35S::OsMADS1-GFP fusions, full-length cDNAs of OsMADS1 and OsMADS1 $1^{l g y 3} 3$ were amplified, and then cloned into p35S::GFPnos vector ${ }^{4}$. The transgenic rice plants were generated by Agrobacterium-mediated transformation as described elsewhere ${ }^{3}$. Relevant primer sequences were given in Supplementary Data 5.

RNA isolation and quantitative PCR. The total RNA was extracted using TRIzol reagent (Invitrogen), and then treated with RNase-free DNase I (Invitrogen) according to the manufacturer's protocol. The RNA was reverse-transcribed using a CDNA synthesis kit (TRANSGEN). Quantitative real-time PCR (qRTPCR) was performed from cDNA using SYBR Green qPCR mix (TRANSGEN, AQ101) following the manufacturer's instructions on an Applied Biosystems 7900 HT Fast Real-Time PCR System. Fold changes were calculated using the $\Delta \mathrm{Ct}$ method, each assay was replicated by at least three time with three biological replicates (independent RNA preparations). The rice Actin3 (LOC_Os03g61970) was used as a reference. Relevant primer sequences were given in Supplementary Data 6.

Yeast two-hybrid assays. Yeast two-hybrid assays were performed as described elsewhere. Full-length cDNAs of DEP1, dep1-1, OsMADS3, OsMADS5, OsMADS7, OsMADS13, OsMADS14, OsMADS15, OsMADS16, and OsMADS55 were amplified and then subcloned into pGBKT7 vector (Takara Bio Inc.), and full-length cDNAs of OsMADS1 and OsMADS1 ${ }^{l g y 3}$ were inserted into pGADT7 (Takara Bio Inc.). All clones were validated by sequencing. The bait and prey vectors were cotransformed into yeast strain AH109, and $\beta$-galactosidase assays were performed according to the manufacturer's protocol (Takara Bio Inc.). The dep1-1 protein was used as a bait to screen a cDNA library from poly(A)-containing RNA isolated from young $(0.2-6 \mathrm{~cm}$ length) panicles. Experimental procedures for screening and plasmid isolation were performed following the manufacturer's instructions. Relevant primer sequences were given in Supplementary Data 5.

SFLC assays. Full-length cDNAs of RGA1, RGB1, RGG1, RGG2, DEP1, dep1-1, GS3, OsGGC2, OsMADS4, OsMADS13, OsMADS14, OsMADS34, OsMADS55, OsMADS58, OsMADS1 $1^{\lg 3}$, and deleted and non-deleted versions of either OsMADS1 or DEP1 were amplified and the amplicons were inserted into pCAMBIA1300-35S-Cluc-RBS or pCAMBIA1300-35S-HA-Nluc-RBS vectors ${ }^{58}$ to generate the required fusion transgenes. Two plasmid vectors for testing the protein-protein interactions (such as DEP1-nLUC and OsMADS1-cLUC), together with the p19 silencing plasmid, were co-transfected into tobacco (N. benthamiana) leaves via Agrobacterium tuiefaciens infiltration, and LUC activity was measured as described elsewher ${ }^{59}$. The injected leaves were detached after $36-48 \mathrm{~h}$ later and sprayed with $1 \mathrm{mM}$ luciferin (Promega, E1605). LUC signal was captured using a cooled CCD-imaging apparatus (Berthold, LB985). Each assay was repeated at least three times. Relevant primer sequences were given in Supplementary Data 5.

Co-immunoprecipitation assays. Full-length cDNAs of DEP1, dep1-1, and OsMADS1 were amplified, and then inserted into either PUC-35S-HA-RBS or PUC-35S-flag-RBS vector ${ }^{31}$. A thaliana protoplasts were transfected with $100 \mu \mathrm{g}$ of plasmid and incubated overnight under low light intensity. Total protein was extracted from harvested protoplasts by treating with $50 \mathrm{mM}$ HEPES (pH7.5), 150 $\mathrm{mM} \mathrm{KCl}, 1 \mathrm{mM}$ EDTA, $0.5 \%$ Trition-X 100, $1 \mathrm{mM}$ DTT, and proteinase inhibitor cocktail (Roche LifeScience). The lysates were incubated with Flag M2 Affinity Gel (Sigma-Aldrich, A2220) at $4{ }^{\circ} \mathrm{C}$ for at least $4 \mathrm{~h}$, then rinsed 5-6 times in the extraction buffer and eluted with $3 \times$ Flag peptide (Sigma-Aldrich, F4709). The immunoprecipitates were electrophoretically separated by SDS-PAGE and transferred to a nitrocellulose membrane (GE Healthcare). Proteins were detected by treating the membranes with anti-HA (1:5,000, MBL, M180-7) or anti-DDDDKtag mAb-HRP-DirectT antibodies (1:10,000, MBL, M185-7). Uncropped blots were shown in Supplementary Fig. 10.

ChIP-PCR assays. ChIP assays were performed as described elsewhere ${ }^{60} .2 \mathrm{~g}$ rice seedlings were grinded with liquid nitrogen and fixed with $1 \%$ formaldehyde under vacuum. After nuclei were isolated and lysed, chromatin was ultrasonic fragmented on ice to an average size of $500 \mathrm{bp}$. The supernatant was firstly blocked with protein A agarose beads pre-absorbed with sheared salmon sperm DNA (Upstate, 16-157), and then a small part of the buffer was setted aside to serve as input. Immunoprecipitations were performed with anti-LGY3 antibody (1:3,000, ABclonal) at $4^{\circ}$ C. At the same time, an equal volume of supernatant was prepared without any antibody for mock sample. Finally, reverse-crosslink and precipitated DNA served as the templates for qRT-PCR. The primer sequences were given in Supplementary Data 6.

EMSA assays. The OsMADS1 lgy3 and OsMADS1 coding sequences were amplified and inserted into pGEX-4T-1 vector (GE Healthcare). GST, GST-OsMADS1, and GST-OsMADS1 19y3 were purified using Glutathione Sepharose 4B (GE Healthcare, 17-0756) following the manufacturer's instructions. DNA probes were amplified and labeled using a biotin label kit (Invitrogen). DNA gel-shift assays were performed using a LightShift Chemiluminescent EMSA Kit (Thermo Fisher Scientific, 20148) as described elsewhere ${ }^{18}$. The relevant primer sequences were given in Supplementary Data 6. 
Transactivation activity assays. The $\sim 2 \mathrm{Kbp}$ DNA fragment of the promoter region of either OsMADS55, OsKANADI24, OsPIN1a, OsARF9, or OsARF14 was amplified from cv. Nipponbare, and used to generate reporter plasmids containing a specific promoter fused to LUC. Full-length cDNAs of OsMADS1, OsMADS1 1 gy 3 , and deleted versions of OsMADS1 were amplified and fused to GAL4BD, and then inserted into pUC19 vector ${ }^{61}$ to generate effector plasmid vectors (e.g., $p G A L B D-O s M A D S 1$ ). The transactivation analysis was performed using rice protoplasts as described elsewhere ${ }^{18}$. The rice protoplasts were transfected with different combinations of vectors overnight, then harvested and lysed for the detection of firefly luciferase activity (Promega, E1960), according to the manufacturer's recommendations. Relevant PCR primer sequences were given in Supplementary Data 5.

Data availability. The authors declare that all relevant data supporting the findings of this study are included in the main manuscript file or Supplementary Information or are available from the corresponding author upon reasonable request.

Received: 21 March 2017 Accepted: 12 January 2018

Published online: 27 February 2018

\section{References}

1. Peng, J. et al. 'Green revolution' genes encode mutant gibberellin response modulators. Nature 400, 256-261 (1999).

2. Sasaki, A. et al. Green revolution: a mutant gibberellin-synthesis gene in rice. Nature 416, 701-702 (2002).

3. Yuan, L. Hybrid rice breeding for super high yield. Hybrid Rice 12, 1-6 (1997).

4. Khush, G. S. Breaking the yield frontier of rice. Geo. J. 35, 329-332 (1995).

5. Wang, S. et al. Control of grain size, shape and quality by OsSPL16 in rice. Nat. Genet. 44, 950-954 (2012).

6. Li, Y. et al. Chalk5 encodes a vacuolar $\mathrm{H}^{+}$-translocating pyrophosphatase influencing grain chalkiness in rice. Nat. Genet. 46, 398-404 (2014).

7. Ashikari, M. et al. Cytokinin oxidase regulates rice grain production. Science 309, 741-745 (2005).

8. Huang, X. et al. Natural variation at the DEP1 locus enhances grain yield in rice. Nat. Genet. 41, 494-497 (2009).

9. Zhou, Y. et al. Deletion in a quantitative trait gene qPE9-1 associated with panicle erectness improves plant architecture during rice domestication. Genetics 183, 315-324 (2009).

10. Sun, $\mathrm{H}$. et al. Heterotrimeric $\mathrm{G}$ proteins regulate nitrogen-use efficiency in rice. Nat. Genet. 46, 652-656 (2014).

11. Wu, Y. et al. The QTL GNP1 encodes GA20ox1, which increases grain number and yield by increasing cytokinin activity in rice panicle meristems. PLoS Genetics 12, e1006386 (2016).

12. Jiao, Y. et al. Regulation of OsSPL14 by OsmiR156 defines ideal plant architecture in rice. Nat. Genet. 42, 541-544 (2010).

13. Miura, K. et al. OsSPL14 promotes panicle branching and higher grain productivity in rice. Nat. Genet. 42, 545-549 (2010).

14. Wang, S. et al. Non-canonical regulation of SPL transcription factors by a human OTUB1-like deubiquitinase defines a new plant type rice associated with higher grain yield. Cell Res. 27, 1142-1156 (2017).

15. Fan, C. et al. GS3, a major QTL for grain length and weight and minor QTL for grain width and thickness in rice, encodes a putative transmembrane protein. Theor. Appl. Genet. 112, 1164-1171 (2006).

16. Ishimaru, K. et al. Loss of function of the IAA-glucose hydrolase gene TGW6 enhances rice grain weight and increases yield. Nat. Genet. 45, 707-711 (2013).

17. Wang, S. et al. The OsSPL16-GW7 regulatory module determines grain shape and simultaneously improves rice yield and grain quality. Nat. Genet. $\mathbf{4 7}$, 949-954 (2015).

18. Wang, Y. et al. Copy number variation at the $G L 7$ locus contributes to grain size diversity in rice. Nat. Genet. 47, 944-948 (2015).

19. Si, L. et al. OsSPL13 controls grain size in cultivated rice. Nat. Genet. $\mathbf{4 8}$, 447-456 (2016).

20. $\mathrm{Hu}$, J. et al. A rare allele of GS2 enhances grain size and grain yield in rice. Mol. Plant 8, 1455-1465 (2015).

21. Che, R. et al. Control of grain size and rice yield by GL2-mediated brassinosteroid responses. Nat. Plants 2, 15195 (2015).

22. Duan, P. et al. Regulation of OsGRF4 by OsmiR396 controls grain size and yield in rice. Nat. Plants 2, 15203 (2015).

23. Song, X. et al. A QTL for rice grain width and weight encodes a previously unknown RING-type E3 ubiquitin ligase. Nat. Genet. 39, 623-630 (2007).

24. Liu, J. et al. GW5 acts in the brassinosteroid signalling pathway to regulate grain width and weight in rice. Nat. Plants 3, 17043 (2017).

25. Duan, P. et al. Natural variation in the promoter of GSE5 contributes to grain size diversity in rice. Mol. Plant 10, 685-694 (2017).
26. Botella, J. R. Can heterotrimeric G proteins help to feed the world? Trends Plant Sci. 17, 563-568 (2012).

27. Sadras, V. O. Evolutionary aspects of the trade-off between seed size and number in crops. Field Crops Res. 100, 125-138 (2007).

28. Dornelas, M. C., Patreze, C. M., Angenent, G. C. \& Immink, R. G. MADS: the missing link between identity and growth? Trends Plant. Sci. 16, 89-97 (2011).

29. Jeon, J. S. et al. leafy hullsterile1 is a homeotic mutation in a rice MADS box gene affecting rice flower development. Plant Cell 12, 871-884 (2000).

30. Parciak, W. Environmental variation in seed number, size and dispersal of a fleshy-fruited plant. Ecology 83, 780-793 (2002).

31. $\mathrm{Hu}, \mathrm{Y}$. et al. Interactions of OsMADS1 with floral homeotic genes in rice flower Development. Mol. Plant 8, 1366-1384 (2015).

32. Prasad, K., Parameswaran, S. \& Vijayraghavan, U. OsMADS1, a rice MADSbox factor, controls differentiation of specific cell types in the lemma and palea and is an early-acting regulator of inner floral organs. Plant. J. 43, 915-928 (2005).

33. Coen, E. S. \& Meyerowitz, E. M. The war of the whorls: genetic interactions controlling flower development. Nature 353, 31-37 (1991).

34. Theissen, G. \& Saedler, H. Plant biology. Flor. Quartets Nat. 409, 469-471 (2001).

35. Henschel, K. et al. Two ancient classes of MIKC-type MADS-box genes are present in the moss Physcomitrella patens. Mol. Biol. Evol. 19, 801-814 (2002).

36. Khanday, I. et al. Genome-wide targets regulated by the OsMADS1 transcription factor reveals its DNA recognition properties. Plant Physiol. 172, 372-388 (2016).

37. Schruff, M. C. et al. The AUXIN RESPONSE FACTOR 2 gene of Arabidopsis links auxin signalling, cell division, and the size of seeds and other organs. Development 133, 251-261 (2006).

38. de Jong, M. et al. Solanum lycopersicum AUXIN RESPONSE FACTOR 9 regulates cell division activity during early tomato fruit development. J. Exp. Bot. 66, 3405-3416 (2015).

39. $\mathrm{Xu}, \mathrm{Q}$. et al. Emerging insights into heterotrimeric $\mathrm{G}$ protein signaling in plants. J. Genet. Genom. 43, 495-502 (2016).

40. Hildebrandt, J. D., Codina, J., Risinger, R. \& Birnbaumer, L. Identification of a gamma subunit associated with the adenylyl cyclase regulatory proteins Ns and Ni. J. Biol. Chem. 259, 2039-2042 (1984).

41. Kato, C. et al. Characterization of heterotrimeric $\mathrm{G}$ protein complexes in rice plasma membrane. Plant J. 38, 320-331 (2004).

42. Robitaille, M. et al. G $\beta \gamma$ is a negative regulator of AP-1 mediated transcription. Cell. Signal. 22, 1254-1266 (2010)

43. Chang, C. W. et al. Acute $\beta$-adrenergic activation triggers nuclear import of histone deacetylase 5 and delays $\mathrm{G}(\mathrm{q})$-induced transcriptional activation. $J$. Biol. Chem. 288, 192-204 (2013).

44. Zhao, M. et al. Regulation of OsmiR156h through alternative polyadenylation improves grain yield in rice. PLoS ONE 10, e0126154 (2015).

45. Urano, D., Chen, J.-G., Botella, J. R. \& Jones, A. M. Heterotrimeric G protein signalling in the plant kingdom. Open Biol. 3, 120186 (2013).

46. Ma, Y. et al. COLD1 confers chilling tolerance in rice. Cell 160, 1209-1221 (2015).

47. Okamoto, H., Matsui, M. \& Deng, X. W. Overexpression of the heterotrimeric G-protein $\alpha$-subunit enhances phytochrome-mediated inhibition of hypocotyl elongation in Arabidopsis. Plant Cell 13, 1639-1652 (2001).

48. Joo, J. H. et al. Different signaling and cell death roles of heterotrimeric G protein $\alpha$ and $\beta$ subunits in the Arabidopsis oxidative stress response to ozone. Plant Cell 17, 957-970 (2005)

49. Huang, J. et al. The plastid protein THYLAKOID FORMATION1 and the plasma membrane G-protein GPA1 interact in a novel sugar-signaling mechanism in Arabidopsis. Plant Cell 18, 1226-1238 (2006).

50. Subramaniam, G. et al. Type B heterotrimeric G protein $\gamma$-subunit regulates auxin and ABA signaling in tomato. Plant Physiol. 170, 1117-1134 (2016).

51. Trusov, Y., Chakravorty, D. \& Botella, J. R. Diversity of heterotrimeric G-protein $\gamma$ subunits in plants. BMC Res. Not. 5, 608 (2012).

52. Choudhury, S. R. \& Pandey, S. Phosphorylation-dependent regulation of G-protein cycle during nodule formation in soybean. Plant Cell 27, 3260-3276 (2015).

53. Bommert, P., Je, B. I., Goldshmidt, A. \& Jackson, D. The maize G $\alpha$ gene COMPACT PLANT2 functions in CLAVATA signalling to control shoot meristem size. Nature 502, 555-558 (2013).

54. De Bodt, S., Raes, J., Van de Peer, Y. \& Theissen, G. And then there were many: MADS goes genomic. Trends Plant Sci. 8, 475-483 (2003).

55. Kaufmann, K., Melzer, R. \& Theissen, G. MIKC-type MADS-domain proteins: structural modularity, protein interactions and network evolution in land plants. Gene 347, 183-198 (2005).

56. Lee, J. H. et al. Regulation of temperature-responsive flowering by MADS-box transcription factor repressors. Science 342, 628-632 (2013). 
57. Posé, D. et al. Temperature-dependent regulation of flowering by antagonistic FLM variants. Nature 503, 414-417 (2013).

58. Chen, H. et al. Firefly luciferase complementation imaging assay for proteinprotein interactions in plants. Plant Physiol. 146, 368-376 (2008).

59. Fujikawa, Y. \& Kato, N. Split luciferase complementation assay to study proteinprotein interactions in Arabidopsis protoplasts. Plant J. 52, 185-195 (2007).

60. Gendrel, A. V., Lippman, Z., Martienssen, R. \& Colot, V. Profiling histone modification patterns in plants using genomic tiling microarrays. Nat. Methods 2, 213-218 (2005).

61. Bart, R. et al. A novel system for gene silencing using siRNAs in rice leaf and stem-derived protoplasts. Plant Methods 2, 13 (2006).

\section{Acknowledgements}

We thank Prof. Nick Harberd for the critical comments on the manuscript. This research was supported by grants from National Natural Science Foundation of China $(91635302$ and 91335207), the National Key Research and Development Program of China

(2016YFD0100401), the Chinese Academy of Sciences (XDA08010101), and the National Special Project of China (2014ZX0800935B).

\section{Author contributions}

Q.L. and R.H. performed most of the experiments; R.H., J.Z., and D.T. conducted the QTL analysis; R.H., K.W., and S.W. constructed the NILs; R.H., Y.W., and Y.P. constructed performed field experiments; J.Z., Q.L., and J.C. performed yeast two-hybrid screen; R.H., K.W., and Y.Y. characterized the phenotype of transgenic plants; K.W. and R.H. analyzed grain quality; and Q.L., X.X., and R.H. were responsible for haplotype analysis. X.F. designed the experiments and wrote the manuscript. All the authors have discussed the results and contributed to the drafting of the manuscript.

\section{Additional information}

Supplementary Information accompanies this paper at https://doi.org/10.1038/s41467018-03047-9.

Competing interests: The authors declare no competing financial interests.

Reprints and permission information is available online at http://npg.nature.com/ reprintsandpermissions/

Publisher's note: Springer Nature remains neutral with regard to jurisdictional claims in published maps and institutional affiliations.

(c) (i) Open Access This article is licensed under a Creative Commons Attribution 4.0 International License, which permits use, sharing, adaptation, distribution and reproduction in any medium or format, as long as you give appropriate credit to the original author(s) and the source, provide a link to the Creative Commons license, and indicate if changes were made. The images or other third party material in this article are included in the article's Creative Commons license, unless indicated otherwise in a credit line to the material. If material is not included in the article's Creative Commons license and your intended use is not permitted by statutory regulation or exceeds the permitted use, you will need to obtain permission directly from the copyright holder. To view a copy of this license, visit http://creativecommons.org/ licenses/by/4.0/.

(C) The Author(s) 2018 